\title{
Mobility of Charge Carriers in Dielectric Liquids
}

\author{
Qingjiang Xue \\ Department of EEE \\ University of Strathclyde \\ Glasgow, United Kingdom \\ qingjiang.xue@strath.ac.uk \\ Mark P Wilson \\ Department of EEE \\ University of Strathclyde \\ Glasgow, United Kingdom \\ mark.p.wilson@strath.ac.uk
}

\author{
Igor Timoshkin \\ Department of EEE \\ University of Strathclyde \\ Glasgow, United Kingdom \\ igor.timoshkin@strath.ac.uk \\ Scott J MacGregor \\ Department of EEE \\ University of Strathclyde \\ Glasgow, United Kingdom \\ scott.macgregor@strath.ac.uk
}

\author{
Martin J Given \\ Department of EEE \\ University of Strathclyde \\ Glasgow, United Kingdom \\ m.given@strath.ac.uk
}

\begin{abstract}
The mobility of charge carriers in dielectric liquids is not only an intrinsic parameter of the conduction process, but it also can define the pre-breakdown processes in the liquids. The charge carrier mobility also affects the electrostatic behavior of fine and ultra-fine (micrometer and sub-micrometer) particles in -fluids, as the charge induced on dispersed particles is defined by the charge concentration and the velocity of charge carriers in the liquid. In this paper, the charge carrier mobility was obtained by measuring conduction current in different dielectric liquids under DC stress. The investigated liquids included traditional mineral oil, synthetic and natural esters. Both, single polarity and polarity reversal methods were used to obtain the charge carrier mobility in these liquids. The results show significant differences in the mobilities of charge carriers in the mineral oil and in ester liquids. Also, the effects of the electric field on the mobilities has been investigated.
\end{abstract}

Keywords - Synthetic esters; mineral oil; electric current; charge carrier mobility

\section{INTRODUCTION}

Traditional mineral oils have been used as insulation liquids in high voltage equipment for more than a century. Apart from the electrical insulation and cooling requirements, the continuous development of electrical power systems has led to new requirements including higher flash point, higher water tolerance, and becoming environmentally friendly. To fulfil these requirements, new types of dielectric liquids, including silicon-based synthetic oil, ester-based synthetic oil, and natural esters, were introduced, and some of them are already in practical use. At the same time, experimental investigation of dielectric properties of these new types of liquids has been ongoing. For example, dielectric performance of mineral oils, synthetic and natural ester liquids, including their breakdown strength, thermal conductivity, and moisture tolerance, was studied in [1], [2].

The mobility of charge carriers plays an important role in understanding of the breakdown behaviour of insulating liquids. This mobility underpins the pre-breakdown processes in insulating liquids. Nano-fluids, produced by the addition of fine and ultra-fine particles to dielectric liquids, have been studied over decades and they are intended to be used as novel insulation liquids. In such liquids, the charge carrier mobility is a key parameter which defines the charging of particles in the host liquids. Information on charge carrier mobility is required for the development of new applications of nanofluids.
In the present work in order to gain a deeper insight into the dielectric behaviour of insulating liquids, the electrical conduction current in three insulation fluids developed for usage in the power industry, MIDEL 7131, MIDEL eN1204, and OM 16 was obtained by different methods. Using these results, the charge carrier mobility was obtained and analysed, reflecting the intrinsic character of these fluids, and laying a foundation for the development and practical applications of novel nano-fluids.

\section{THEORY OF CHARgE CARRIER MOBILITY}

Insulation fluids consists of nonpolar or weakly-polar molecules, and hence can be considered as weak electrolytes. When stressed with an external electric field, ions can be created by ionic pair dissociations in the bulk of liquid, or by charge injection mechanisms near the metal-liquid interfaces. These ions start to migrate in the applied field due to the electrostatic forces, which is the main mechanism responsible for electrical conduction in insulation oils, [3]. It is difficult to describe accurately all processes contributing to conduction in insulating oils and ester liquids, therefore the apparent charge carrier mobility, based on the ion drift model, is widely used to investigate the conduction behaviour of such dielectric liquids [4].

When the liquid is stressed with a DC voltage, the ions in the bulk of liquid are accelerated and after a relaxation process start to move with a constant drift velocity. The drift velocity, $v_{\text {drift }}$, can be expressed using the ion mobility, $\mu$, and electric field strength, $E$, as:

$$
v_{\text {drift }}=\mu E
$$

Based on (1), the mobility of the charge carriers can then be obtained by measuring the time of flight (TOF): once the liquid was stressed with a step DC voltage, the charge carriers existing in the liquid would start to migrate to the electrodes, leading to a transient current. A single current peak is observed if the charge carriers are similar in nature. The time interval between the application of voltage and the occurrence of the current peak is defined as the TOF. With TOF, the velocity of the charge carriers can be calculated as $v_{\text {drift }}=d / T O F$, where $d$ is the distance between the two electrodes. Thus, the mobility can be derived as: 


$$
\mu=\frac{v_{d r i f t}}{E}=\frac{d^{2}}{T O F \cdot U}
$$

where $U$ is the magnitude of the applied voltage.

In the practical measurements of mobility, two techniques are widely used: the single polarity method and the polarity reversal method. For the single polarity method, a step voltage is applied to the liquids. The current is then measured to determine the TOF. For the polarity reversal method, a step voltage is applied for a certain time and then immediately reversed to the opposite polarity. In the single polarity method, charge carriers need a long time (a few hours) to relax to the thermodynamic equilibrium after removal of the electric stress, which is not the case with the polarity reversal method, [5].

\section{EXPERIMENT DETAILS}

\section{A. The Investigated Liquids}

Three types of insulation liquids were investigated in this work: MIDEL 7131, MIDEL eN1204, and OM 16. MIDEL 7131 is a synthetic ester, and MIDEL eN1204 is a natural ester. OM 16 is a traditional mineral insulation oil. The basic parameters of the liquids are listed in Table I.

TABLE I. PhysicAl AND ElectricAl Properties of Tested DIELECTRIC LIQUIDS, [6], [7].

\begin{tabular}{|l|c|c|c|}
\hline \multirow{2}{*}{\multicolumn{1}{|c|}{ Parameters }} & \multicolumn{3}{|c|}{ Dielectric Liquids } \\
\cline { 2 - 4 } & $\begin{array}{c}\text { MIDEL } \\
\mathbf{7 1 3 1}\end{array}$ & $\begin{array}{c}\text { MIDEL } \\
\text { eN1204 }\end{array}$ & OM 16 \\
\hline Dielectric Constant & 3.3 & 3.3 & 2.3 \\
\hline $\begin{array}{l}\text { Electrical } \\
\text { Conductivity }\left(\mathrm{pS} / \mathrm{m}^{2}\right)\end{array}$ & 4.4 & 5 & 0.061 \\
\hline $\begin{array}{l}\text { Density } \\
\left(\mathrm{g} / \mathrm{mL}, 20{ }^{\circ} \mathrm{C}\right)\end{array}$ & 0.97 & 0.92 & 0.89 \\
\hline $\begin{array}{l}\text { Viscosity } \\
\left(\mathrm{mm} / \mathrm{sec}, 20{ }^{\circ} \mathrm{C}\right)\end{array}$ & 70 & 85 & 26 \\
\hline
\end{tabular}

\section{B. Test Cell}

Two test cells were used in the experiments to conduct the tests with different electric field strengths. The first one was a stainless-steel coaxial cylindrical test cell, whose crosssectional view is shown in Fig. 1. (a).
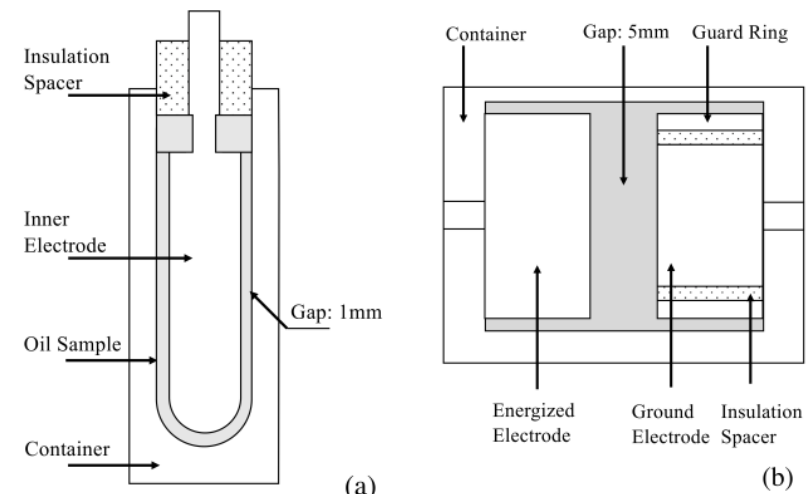

(a)

(b)
The second test cell consisted of two parallel electrodes, as shown in Fig. 1. (b). The two electrodes were made of polished brass, and the ground electrode was equipped with a guard ring. Compared to the first test cell, the longer electrode distance enabled a much higher voltage to be applied during the experiment. Besides, this test cell can also be used to measure the behavior of nano-fluids, as the short interelectrode distance of the first test cell increases the probability of breakdown due to the particles inside, which provides an avenue for future work.

\section{Measurement System}

The conduction current in the liquids was measured by a Keithley 6514 electrometer, which was controlled by LabVIEW software. A built-in repeating filter was set up to obtain accurate measurements: three direct readings were collected and then averaged to yield one reading, which the software received and stored. This process took $0.5 \mathrm{~s}$, allowing a sampling rate of two data per second for the electric current measurements.

Two different types of power supply were applied in these experiments: a Matsusada Precision AU-60 power supply, controlled by LabVIEW via a NI DAQ card to output a DC voltage up to $60 \mathrm{kV}$, either positive or negative. A Keithley 617 electrometer, controlled via a GPIB card, was also used to provide a $\mathrm{DC}$ voltage ranging from $-102 \mathrm{~V}$ to $+102 \mathrm{~V}$ in $50 \mathrm{mV}$ increments.

\section{Measurement Procedure}

Each individual test was conducted on a fresh sample of each liquid after cleaning of the electrodes and test cell body with ethanol and lint-free tissue. All tests were conducted in triplicate.

The coaxial test cell was used with the reverse polarity method: a negative step voltage was applied to the coaxial test cell for $30 \mathrm{~min}$, then the voltage polarity was reversed to positive and the test continued for a further $30 \mathrm{~min}(60 \mathrm{~min}$ total). The TOF was measured according to the time of occurrence of the current peak. Three voltage levels were selected for this test: $40 \mathrm{~V}, 60 \mathrm{~V}$, and $80 \mathrm{~V}$.

Due to the limitation from the significantly higher voltages applied in the tests with the guard ring test cell, the single polarity method was used. Any residual effect induced by the previous test with this method was avoided by testing a fresh liquid sample for each measurement and carefully cleaning the test cell with ethanol and lint-free tissue between measurements. Six voltages: $\pm 2 \mathrm{kV}, \pm 4 \mathrm{kV}$, and $\pm 6 \mathrm{kV}$ were selected and each of them was applied for $60 \mathrm{~min}$ to determine the TOF.

\section{RESULTS AND DISCUSSION}

\section{A. Polarity Reversal Method}

Samples of all three liquids were tested in the coaxial test cell using the polarity reversal method. The results showed that the TOFs of the liquids were distributed within $100 \mathrm{~s}$, yielding less than 200 data-points. The original current data were filtered by a Savitzky-Golay (S-G) filter to avoid the random noise before the determination of TOF value. Fig. 2.(a) and Fig. 2. (b) shows the filtered current and the mobility obtained from these filtered data respectively.

Fig. 1. (a), Cross-sectional side view of the coaxial test cell; (b), Crosssectional side view of the parallel electrode test cell. 
From Fig. 2. (a), it can be found that the current in all three liquids increased with an increase in the electric field strength. The obtained mobility for the mineral oil is similar to the mobility values reported previously: $\sim 10^{-9} \mathrm{~m}^{2} / \mathrm{V} \cdot \mathrm{s},[9]$, and $\sim\left(10^{-10}-10^{-9}\right) \mathrm{m}^{2} / \mathrm{V} \cdot \mathrm{s},[10]$. The current in the synthetic ester and the natural ester is almost one order of magnitude higher than that in the mineral oil, indicating the higher electrical conductivity of both synthetic and natural esters.
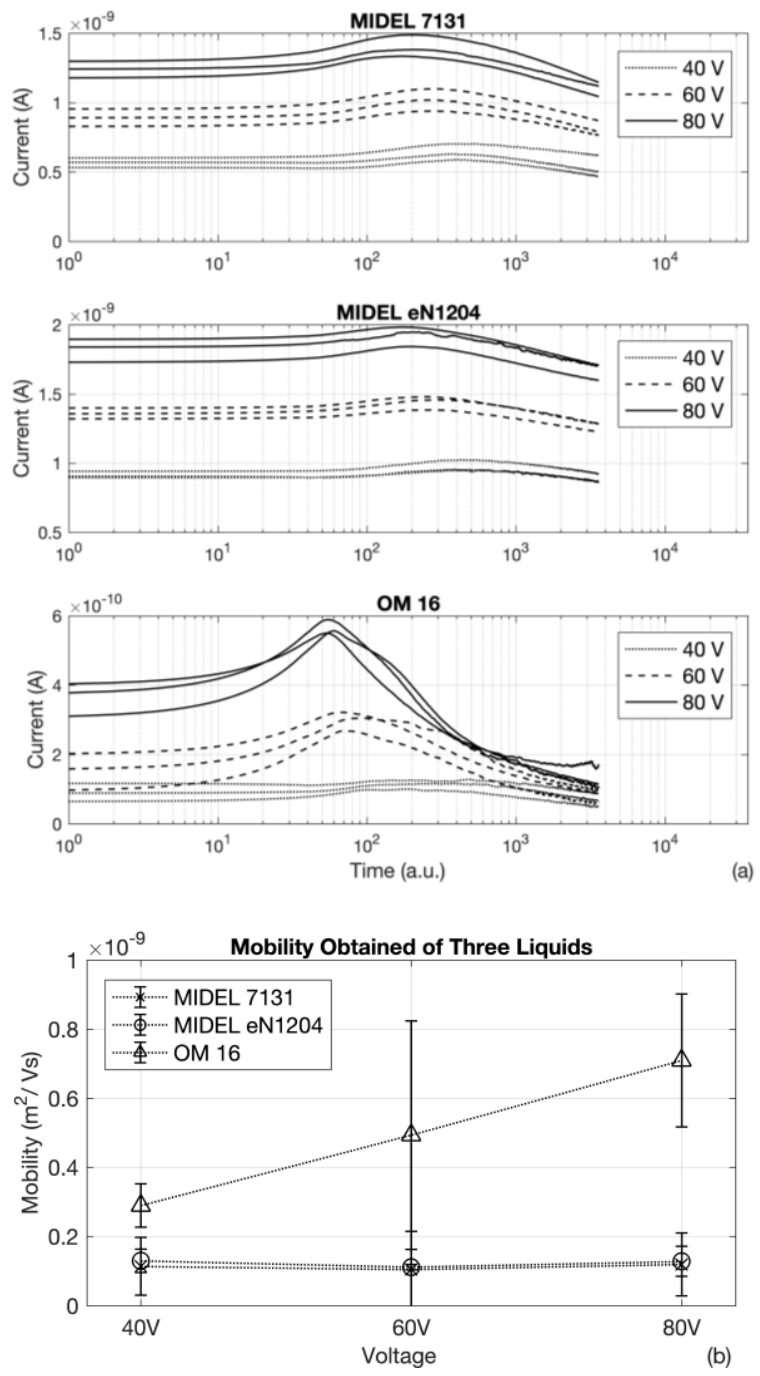

Fig. 2. (a), Current as a function of time for different voltages (one a.u. corresponds to $0.5 \mathrm{~s}$ ); (b), Mobilities for all tested liquids, error bars present $95 \%$ confidence intervals.

Regarding the mobility presented in Fig. 2. (b), it can be seen that the mobility of charge carriers in OM 16 increased with the field strength, while the mobility for the other two liquids remained constant. The increase of the obtained mobility in the OM 16 could be attributed to the electrohydrodynamic (EHD) effect, [8], as the OM 16 has significantly lower viscosity than the other two liquids. The mobility obtained in the present work is hence more affected by the EHD velocity of the liquids, which leads the measured mobility being greater than the mobility associated with charge carriers.

The relationship between the conductivity and charge carrier mobility is reviewed firstly. Given a dielectric liquid under DC stress, the current density $J$ can be given as a function of charge carrier mobility and field strength:

$$
J=n \cdot \mu \cdot E
$$

where $n$ is the charge density in the liquid. Current density can also be expressed as:

$$
J=\sigma \cdot E
$$

Thus, the electrical conductivity can be expressed as:

$$
\sigma=n \cdot \mu
$$

From Fig. 2. (a), it can be seen that the electric current in all the three liquids increased similarly with voltage, showing a nearly-constant electrical conductivity in these three liquids. Combining this conclusion with the obtained mobilities (excluding the enhancement from the EHD effect to samples of OM 16, the mobility of the three liquids should be of the same order) and equation (5), it can be concluded that the charge carrier density in synthetic and natural esters is nearly one order of magnitude higher than the charge carrier density in the OM 16. This is in agreement with the fact that the ester liquids consist of weakly-polar molecules, while the mineral oils consist of nonpolar molecules, [9].

\section{B. Single Polarity Method}

As discussed previously, the single polarity method requires a long waiting time to complete each test. By using a fresh sample of liquid in each test in this experiment, the single polarity method showed a good repeatability in the current measurements. Fig. 3 shows the filtered current as a function of time for the three liquids.

Compared with the synthetic and natural esters, the TOF in mineral oil is very short ( $2 \mathrm{~s}$ to $3 \mathrm{~s})$. As the data collection rate of the electrometer is only 2 readings per second, the effect on the TOF of different applied voltages is difficult to determine. It could only be calculated that the charge carrier mobility of samples of OM 16 obtained in this test was between $10^{-9} \mathrm{~m}^{2} / \mathrm{V} \cdot \mathrm{s}$ and $10^{-8} \mathrm{~m}^{2} / \mathrm{V} \cdot \mathrm{s}$.

It should also be clarified that the larger inter-electrode spacing and the significantly higher electric field in the parallel electrode test cell enhanced the EHD effect significantly; the enhanced motion of the liquids could be observed directly, which indicates that the mobility obtained in these tests was dominated by the fluids motion.

The synthetic and natural esters have the higher viscosity that the mineral oil, and the TOFs of these two liquids were longer than those for mineral oil, making it possible to calculate and compare mobilities under different voltages, as shown in Fig. 4. Moreover, the second current peak was observed in both types of ester liquids, synthetic and natural. The mobilities related to the second current peak are also included in Fig. 4. It can be found that the mobility extracted from the second peak is significantly lower than the mobility related to the first peak. This may indicate that slower charge carriers were responsible for these lower mobility values.

Thus, these results demonstrate that there are lower mobility charge carriers in the synthetic and natural esters which may be associated with the fact that these liquids have a higher viscosity, as compared with the mineral oil. However, this still needs confirmation via future work. 

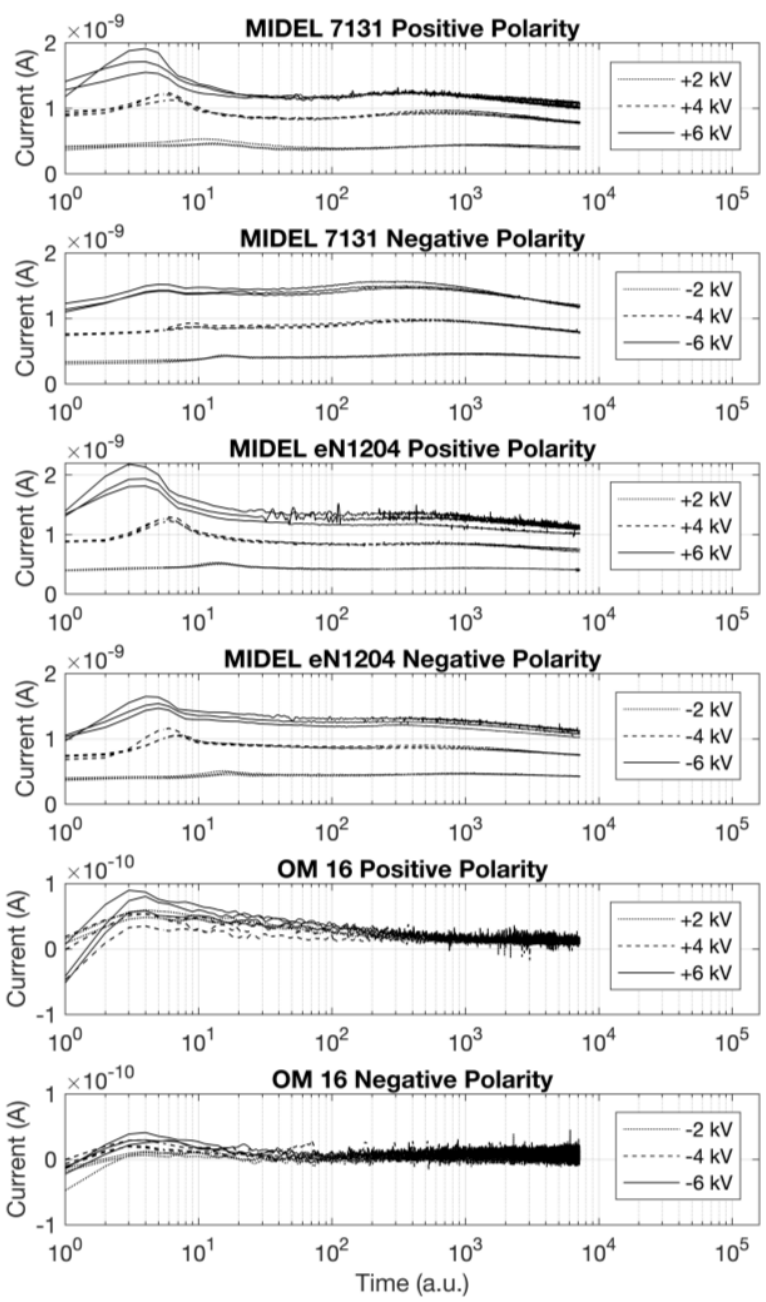

Fig. 3. Current as a function of time for different voltages. One a.u. corresponds to $0.5 \mathrm{~s}$.
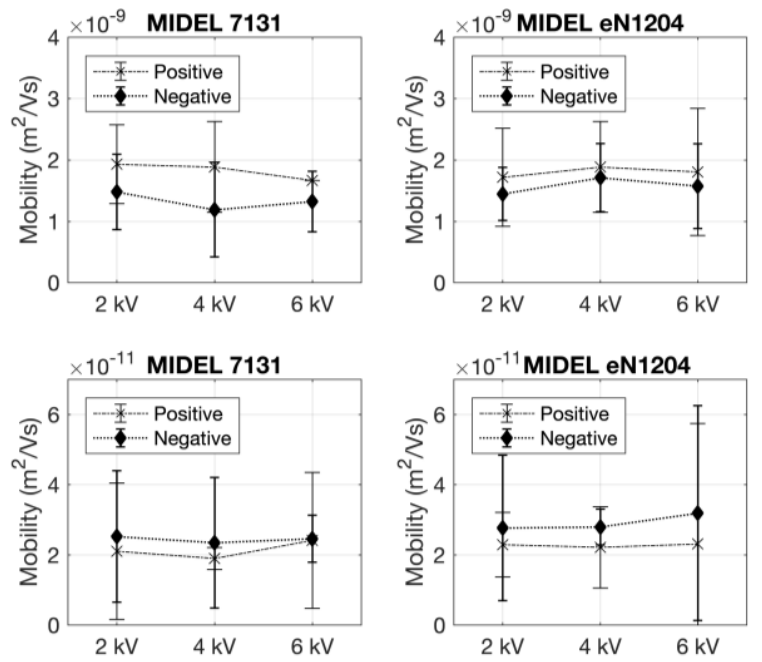

Fig. 4. The mobilities obtained for MIDEL 7131 and MIDEL eN1204. The $1^{\text {st }}$ row are the mobilities obtained from the first current peak while the $2^{\text {nd }}$ row are the mobilities obtained from the second current peak. Error bars show $95 \%$ confidence intervals.

\section{CONCLUSIONS}

Samples of three different dielectric liquids were tested with two methods to investigate their electric characteristics, in the form of the charge carrier mobility. The results from the polarity reversal tests reflected lower charge carrier density in the mineral oil than in the other two liquids. By changing samples between tests, the single polarity method was successfully practiced with stronger applied electrical field, and dual TOFs were found in the synthetic and natural esters, which could provide an avenue for future investigation.

\section{REFERENCES}

[1] A. Beroual, U. Khaled, P. S. M. Noah, and H. Sitorus, "Comparative study of breakdown voltage of mineral, synthetic and natural oils and based mineral oil mixtures under AC and DC voltages," Energies, vol. 10, no. 4, pp. 1-17, 2017.

[2] K. B. Liland, D. Linhjell, C. Lesaint, M. H. Glomm Ese, L. E. Lundgaard, P. Mavrommatis, and N. Stjepanovic, "Comparison between synthetic ester and mineral oil impregnated transformer paper aging markers," IEEE 19th Int. Conf. Dielectr. Liquids, ICDL 2017, 2017, pp. 1-6.

[3] A. Alj, A. Denat, J. P. Gosse, B. Gosse, and I. Nakamura, "Creation of Charge Carriers in Nonpolar Liquids,” IEEE Trans. Electr. Insul., vol. 20, no. 2, pp. 221-231, 1985.

[4] U. Gafvert, A. Jaksts, C. TÖrnkvist, and L. Walfridsson, "Electrical Field Distribution in Transformer Oil," IEEE Trans. Electr. Insul., vol. 2, no. 3, pp. 647-660, 1992.

[5] L. Yang, M. S. Zadeh, J. Schiessling, O. Hjortstam, Y. V. Serdyuk, and S. M. Gubanski, "Measurement of ion mobility in transformer oils for HVDC applications,” Int. Conf. High Volt. Eng. Appl., ICHVE 2012, pp. $464-467,2012$.

[6] MInistry of Defence, Standard 01-5 Fuels, Lubricants and Associated Products, no. 13. 2002.

[7] M\&I MATERIALS, "MIDEL eN 1204 Technical Information Pack," 2016

[8] M\&I MATERIALS, "Midel 7131 Safety Data Sheet," 2018.

[9] L. Yang, S. Gubanski, Y. V. Serdyuk, and J. Schiessling, "Dielectric properties of transformer oils for HVDC applications," IEEE Trans. Dielectr. Electr. Insul., vol. 19, no. 6, pp. 1926-1933, 2012.

[10] Y. Lv, Q. Du, L. Wang, Q. Sun, M. Huang, C. Li, B. Qi, Y. Lv, Q. Du, L. Wang, Q. Sun, and M. Huang, "Effect of $\mathrm{TiO}_{2}$ nanoparticles on the ion mobilities in transformer oil-based nanofluid," AIP Adv., vol.7, $105022,2017$.

[11] P. Atten, "Electrohydrodynamic instability and motion induced by injected space charge in insulating liquids," IEEE Trans. Dielectr. Electr. Insul., vol. 3, no. 1, pp. 1-17, 1996.

[12] Y. Wang, F. Wang, J. Li, S. Liang, and J. Zhou, "Electronic Properties of Typical Molecules and the Discharge Mechanism of Vegetable and Mineral Insulating Oils,” Energies, vol. 11, no. 3, p. 523, 2018. 